\title{
The Solution of the Kato Problem for Divergence Form Elliptic Operators with Gaussian Heat Kernel Bounds
}

\author{
Steve Hofmann* \\ Department of Mathematics \\ University of Missouri-Columbia \\ Columbia, MO 65211 \\ e-mail: hofmann@math.missouri.edu \\ Michael Lacey ${ }^{\dagger}$ \\ School of Mathematics \\ Georgia Institute of Technology \\ Atlanta, GA 30332 \\ email: lacey@math.gatech.edu \\ Alan McIntosh ${ }^{\ddagger}$ \\ Centre for Mathematics and its Applications \\ Australian National University \\ Canberra, ACT 0200 Australia \\ email: alan@maths.anu.edu.au \\ February 1, 2001
}

*supported by NSF

$\dagger_{\text {supported by NSF }}$

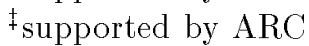




\section{Introduction, history, and statement of the Main Theorem}

Let $A$ be an $n \times n$ matrix of complex, $L^{\infty}$ coefficients, defined on $\mathbb{R}^{n}$, and satisfying the ellipticity (or "accretivity") condition

$$
\lambda|\xi|^{2} \leq \operatorname{Re}\langle A \xi, \xi\rangle \equiv \operatorname{Re} \sum_{i, j} A_{i j}(x) \xi_{j} \bar{\xi}_{i},\|A\|_{\infty} \leq \Lambda
$$

for $\xi \in \mathbb{C}^{n}$ and for some $\lambda, \Lambda$ such that $0<\lambda \leq \Lambda<\infty$. We define a divergence form operator

$$
L u \equiv-\operatorname{div}(A(x) \nabla u)
$$

which we interpret in the usual weak sense via a sesquilinear form.

The accretivity condition (1.1) enables one to define an accretive square root $\sqrt{L} \equiv L^{1 / 2}$ (see [12]), and a fundamental issue is to "solve the square root problem", i.e. to establish the estimate

$$
\|\sqrt{L} f\|_{L^{2}\left(\mathbb{R}^{n}\right)} \leq C\|\nabla f\|_{L^{2}\left(\mathbb{R}^{n}\right)},
$$

with $C$ depending only on $n, \lambda$ and $\Lambda$. The latter estimate is connected with the question of the analyticity of the mapping $A \rightarrow L^{\frac{1}{2}}$, which in turn has applications to the perturbation theory for certain classes of hyperbolic equations (see [15]). We remark that (1.3) is equivalent to the opposite inequality for the square root of the adjoint operator $L^{*}$.

In $[12,13]$, Kato conjectured that an abstract version of (1.3) might hold, for "regularly accretive operators" (see [13, 17] for the details). A counterexample to this abstract conjecture was obtained by McIntosh [16], who then reformulated the conjecture in the following form, bearing in mind that Kato's interest in the problem had been motivated by the special case of elliptic differential operators.

Conjecture 1.4. The estimate (1.3) holds, for L defined as in (1.2), for any $L^{\infty}, n \times n$ matrix A with complex entries, for which (1.1) holds.

To establish the validity of Conjecture 1.4 has become known as the "Kato Problem", or "square root problem". Until recently, it had been solved completely only in one dimension [5], where it is essentially equivalent to the 
problem of proving the $L^{2}$ boundedness of the Cauchy integral operator on a Lipschitz curve (see also [14]).

For $n>1$, a restricted version of Conjecture 1.4, also essentially posed by Kato in [13], has recently been proved by one of us (Hofmann), along with Auscher, Lewis, and Tchamitchian in [10]. The restricted version treats the case that $A$ is close, in the $L^{\infty}$ norm, to a real symmetric matrix of bounded, measurable coefficients. It is this version that yields the perturbation results for hyperbolic equations alluded to above (see [15]).

Prior to the latter result, Conjecture 1.4 had been proved in higher dimensions in the case that $A$ is close to a constant matrix, in either the $L^{\infty}$ norm ([5], [8], [9], [11]), or the BMO norm ([7], [4]).

In the present paper, we solve the "Kato problem", i.e. we establish the validity of Conjecture 1.4, in the case that the heat kernel of the operator $L$ satisfies the "Gaussian" property, which we define as follows. Let $W_{t^{2}}(x, y)$ denote the kernel of the operator $e^{-t^{2} L}$. We say that $L$ satisfies "property $(\mathrm{G})$ " if there exist positive constants $\alpha$ and $\beta$ such that

$$
\begin{aligned}
& \text { (i) }\left|W_{t^{2}}(x, y)\right| \leq \beta t^{-2} \exp \left\{\frac{-|x-y|^{2}}{\beta t^{2}}\right\} \\
& \text { (ii) }\left|W_{t^{2}}(x+h, y)-W_{t^{2}}(x, y)\right|+\left|W_{t^{2}}(x, y+h)-W_{t^{2}}(x, y)\right| \\
& \leq \beta \frac{|h|^{\alpha}}{t^{\alpha+2}} \exp \left\{\frac{-|x-y|^{2}}{\beta t^{2}}\right\},
\end{aligned}
$$

where the latter inequality holds whenever either $|h| \leq t$, or $|h| \leq|x-y| / 2$.

We remark that by [3], property $(G)$ always holds in two dimensions, and also in an $L^{\infty}$ neighborhood of any real, symmetric matrix, by the classical parabolic regularity theory of Nash-Moser-Aronson, combined with a perturbation result of [1] (or [4, Chapter 1.2, Theorem 6(ii)]). In each of these cases, $\alpha$ and $\beta$ may be taken to depend only on $n, \lambda$ and $\Lambda$.

Our main result is the following:

Theorem 1.5. Suppose that $A$ is an $n \times n$ matrix of $L^{\infty}$, complex coefficients, defined on $\mathbb{R}^{n}$, such that (1.1) holds, and let $L \equiv-\operatorname{div}(A(x) \nabla)$. We suppose also that $L$ satisfies property $(G)$. Then the square root estimate (1.3) holds, with $C \equiv C(\alpha, \beta, n, \lambda, \Lambda)$.

In particular, this theorem solves Conjecture 1.4 completely in two dimensions, and also recaptures the result of [10], treating the restricted version of the conjecture, in all dimensions. 
We further remark that the Gaussian hypothesis may be removed, thus resulting in the complete solution to the Kato problem for second order divergence form operators. We shall do this jointly with P. Auscher and P. Tchamitchian, in a second paper [2].

Acknowledgments This collaboration began while the third author was at Macquarie University, and the first author visited him there in 1996. It was completed during the second author's visit to ANU in Canberra. We thank Pascal Auscher and Philippe Tchamitchian for numerous interesting and useful conversations concerning the problem, and in particular, for describing to us their joint work [4].

\section{Preliminary Arguments}

We begin by introducing some notation that we shall use throughout the sequel. The generic constant $C$, which may vary from one place to the next, is allowed to depend upon $\alpha, \beta, n, \lambda$ and $\Lambda$, which five parameters we shall henceforth refer to as "allowable". When a constant depends on other quantities, we shall note that dependence explicitly. Given a cube $Q \in \mathbb{R}^{n}$, let $\ell(Q)$ denote the side length of $Q$, and let $k Q$ denote the concentric dilate of $Q$, having side length $k \ell(Q)$. We also let $R_{Q}$ denote the Carleson box $R_{Q} \equiv Q \times(0, \ell(Q))$. We set $\varphi(x)=x$. In the sequel, $\varphi$ will always denote this function.

We shall deduce Theorem 1.5 as a consequence of a modified version of a "Tb Theorem for square roots" proved by Auscher and Tchamitchian [4]. To this end, we define a family $\left\{\gamma_{t}\right\}_{t=0}^{\infty}$ of mappings $\gamma_{t}: \mathbb{R}^{n} \rightarrow \mathbb{C}^{n}$ by

$$
\gamma_{t}(x) \equiv e^{-t^{2} L} t L \varphi \equiv-e^{-t^{2} L} t \operatorname{div} A
$$

since the Jacobian matrix $\nabla \varphi \equiv \mathbf{1}$, the $n \times n$ identity matrix. It is a routine matter to deduce from property $(G)$ that

$$
C_{0} \equiv \sup _{t>0}\left(\left\|\gamma_{t}(\cdot)\right\|_{L^{\infty}\left(\mathbb{R}^{n}\right)}+t^{\alpha} \sup _{h \neq 0}\left\||h|^{-\alpha}\left(\gamma_{t}(\cdot+h)-\gamma_{t}(\cdot)\right)\right\|_{L^{\infty}\left(\mathbb{R}^{n}\right)}\right)^{2}<\infty,
$$

and moreover $C_{0}$ depends only on the allowable parameters. We omit the details. 
Now let us describe the " $T b$ Theorem for square roots" of [4], or rather our simple modification of it, to which we alluded above. Let $\mathbf{J}$ denote an index set with finite cardinality $N$. Suppose that there exist constants $C^{\prime}$ and $C^{\prime \prime}$, such that for each cube $Q \subseteq \mathbb{R}^{n}$, there is a family $\left\{F_{\nu}\right\}, \nu \in \mathbf{J}$, of functions $F_{\nu} \equiv F_{\nu, Q}: 5 Q \rightarrow \mathbb{C}$, which satisfy

$$
\begin{aligned}
\text { (i) } \quad & \int_{5 Q}\left|\nabla F_{\nu}\right|^{2} \leq C^{\prime}|Q| \\
\text { (ii) } \quad & \int_{5 Q}\left|L F_{\nu}\right|^{2} \leq C^{\prime \prime} \frac{|Q|}{(\ell(Q))^{2}} \\
\text { (iii) } \quad & \sup _{Q} \frac{1}{|Q|} \int_{Q} \int_{0}^{\ell(Q)}\left|\gamma_{t}(x)\right|^{2} \frac{d t}{t} d x \leq \\
& C \sum_{\nu \in \mathbf{J}}\left\{C_{0}+\sup _{Q} \frac{1}{|Q|} \int_{Q} \int_{0}^{\ell(Q)}\left|\gamma_{t}(x) P_{t}\left(\nabla F_{\nu}\right)(x)\right|^{2} \frac{d t}{t} d x\right\},
\end{aligned}
$$

where $P_{t}$ denotes a nice approximate identity, given by convolution with a function $t^{-n} P(x / t) \equiv P_{t}(x) \in C_{0}^{\infty}$, with $0 \leq P(x) \leq 1, \operatorname{supp} P_{t}(x) \subseteq$ $B_{t}(0) \equiv\left\{x \in \mathbb{R}^{n}:|x|<t\right\}$, and $\int P(x) d x=1$. We then have the following modification of a result of [4], Section 3.2.

Theorem 2.4. Suppose that A, $L$ are as in Theorem 1.5, and that there is a finite index set $\mathbf{J}$, with cardinality $N$, such that for each cube $Q \subseteq \mathbb{R}^{n}$, there exist functions $F_{\nu} \equiv F_{\nu, Q}: 5 Q \rightarrow \mathbb{C}$, indexed by $\mathbf{J}$, and satisfying (2.3). Then the square root estimate (1.3) holds for $L^{\frac{1}{2}}$, with a bound on the order of $C\left(1+\sqrt{N\left(C_{0}+C^{\prime}+C^{\prime \prime}\right)}\right)$.

Thus, to prove Theorem 1.5, it suffices to construct, for each Q, a family $F_{\nu} \equiv F_{\nu, Q}$, with $\nu$ ranging over some finite index set $\mathbf{J}$ of fixed cardinality, satisfying (2.3). It is this construction which is the contribution of the present work.

We note that in [4], Theorem 2.4 is proved in the case that $N=1$, and for a $\mathbb{C}^{n}$-valued version of $F$. The proof of our version is essentially identical to that of [4], and we omit it. We remark that the original version does yield a proof of Theorem 1.5 in 2 dimensions, by a variant of the methods of the present paper, and this was actually done first. In extending matters to dimensions $n>2$, it is helpful to deal with scalar $F$. The present formulation of Theorem 2.4 is useful for this purpose. In the next section, we construct the family $F_{\nu, Q}$, and verify that it has the required properties. 


\section{Proof of Theorem 1.5: construction of $F_{\nu}$}

Given $Q$, with side length $\ell(Q) \equiv \rho$, we define a mapping $F \equiv F_{Q}: 5 Q \rightarrow \mathbb{C}^{n}$, from which we shall construct the functions $F_{\nu} \equiv F_{\nu, Q}$ required by Theorem 2.4. We recall that $\varphi(x) \equiv x$. Let $\epsilon>0$ be a small number to be chosen. We define $F \equiv F_{Q}$ to be

$$
F \equiv e^{-\epsilon^{2} \rho^{2} L} \varphi .
$$

Given a family $\{\nu\}$ of unit vectors in $\mathbb{C}^{n}$, we define

$$
F_{\nu} \equiv F \cdot \bar{\nu} .
$$

Using "Property $(\mathrm{G})$ ", the reader may readily verify that each $F_{\nu}$ satisfies (2.3)(i) and (ii), and we omit the routine details (although we do point out that, to prove (i), one mimics the proof of Cacciopoli's inequality; estimate (ii) is even easier). We observe that the constant in (2.3)(ii) is on the order of $C \epsilon^{-2}$, but as we shall eventually choose $\epsilon$ to depend only upon the allowable parameters, this will be harmless. We emphasize, however, that the constant in (2.3)(i) is independent of $\epsilon$, and this fact is important. The main point, of course, is to verify that (2.3)(iii) holds for some finite collection of unit vectors $\{\nu\}$, and it is to this task that we now turn our attention.

We consider cones

$$
C=C_{\nu} \equiv\left\{z \in \mathbb{C}^{n}:|z-\nu(z \cdot \bar{\nu})|<\epsilon|z \cdot \bar{\nu}|\right\},
$$

in which $\nu$ is a complex vector of modulus one. Clearly, we may select a set of complex unit vectors $\nu$, with cardinality $N=N(\epsilon, n)$, such that the corresponding cones $C_{\nu}$ cover $\mathbb{C}^{n}$. It is then enough to show that the following analogue of (2.3)(iii) holds in each cone separately:

$$
\begin{aligned}
& \sup _{Q} \frac{1}{|Q|} \int_{Q} \int_{0}^{\ell(Q)}\left|\gamma_{t}(x) 1_{C_{\nu}}\left(\gamma_{t}(x)\right)\right|^{2} \frac{d t}{t} d x \leq \\
& C\left\{C_{0}+\sup _{Q} \frac{1}{|Q|} \int_{Q} \int_{0}^{\ell(Q)}\left|\gamma_{t}(x) P_{t}\left(\nabla F_{\nu}\right)(x)\right|^{2} \frac{d t}{t} d x\right\} .
\end{aligned}
$$

Moreover, by a well known reduction (see, e.g., [10], Lemma 3.3), it suffices to prove a version of the latter estimate on an appropriately ample sawtooth domain. To be precise, it is enough to show that there is a positive number $\eta$, 
depending only on the allowable parameters, such that for each cube $Q$, and each fixed unit vector $\nu \in \mathbb{C}^{n}$, we have a collection $\left\{Q_{k}\right\}$ of non-overlapping dyadic subcubes of $Q$, and a decomposition $Q=E \cup B$, with $E \cap B=\emptyset$, $|E| \geq \eta|Q|$, and $B=\cup Q_{k}$, for which

$$
\begin{aligned}
& \iint_{E^{*}}\left|\gamma_{t}(x) 1_{C_{\nu}}\left(\gamma_{t}(x)\right)\right|^{2} \frac{d t}{t} d x \leq \\
& C\left\{C_{0}|Q|+\int_{Q} \int_{0}^{\ell(Q)}\left|\gamma_{t}(x) P_{t}\left(\nabla F_{\nu}\right)(x)\right|^{2} \frac{d t}{t} d x\right\},
\end{aligned}
$$

where $E^{*} \equiv R_{Q} \backslash\left(\cup R_{Q_{k}}\right)$.

To this end, we fix $Q$, and a unit vector $\nu \in \mathbb{C}^{n}$, and we define $F$ as in (3.1). We recall that $F_{\nu} \equiv F \cdot \bar{\nu}$. We begin by observing that since $\varphi$ is Lipschitz, we have that

$$
\|F-\varphi\|_{\infty} \leq C \epsilon \rho
$$

Indeed, $F-\varphi \equiv\left(e^{-\epsilon^{2} \rho^{2} L}-I\right) \varphi$, and we may then obtain estimate (3.3) as an easy consequence of Property $(\mathrm{G})$ and the fact that $e^{-\epsilon^{2} \rho^{2} L} 1=1$. We omit the routine details. It follows immediately from (3.3) and an integration that

$$
|Q|^{-1}\left|\int_{Q}(\nabla F-\mathbf{1}) d x\right| \leq C \epsilon,
$$

were $\mathbf{1} \equiv \nabla \varphi$ is the $n \times n$ identity matrix. Consequently,

$$
|Q|^{-1}\left|\int_{Q}\left(\nu \cdot \nabla F_{\nu}-1\right) d x\right| \leq C \epsilon .
$$

Let $\mathbf{S}_{1}$ denote the set of maximal dyadic subcubes $Q^{\prime} \subseteq Q$ for which

$$
\left|Q^{\prime}\right|^{-1} \operatorname{Re} \int_{Q^{\prime}} \nu \cdot \nabla F_{\nu} d x \leq 3 / 4,
$$

and let $B_{1}$ denote the union of these cubes. Take $\mathbf{S}_{2}$ to be the set of maximal dyadic subcubes $Q^{\prime} \subseteq Q$ such that

$$
\left|Q^{\prime}\right|^{-1} \int_{Q^{\prime}}\left|\nabla F_{\nu}\right| d x>(8 \epsilon)^{-1}
$$


and let $B_{2}$ denote the union of the cubes in $\mathbf{S}_{2}$. We set $B \equiv B_{1} \cup B_{2}$, and $E \equiv Q \backslash B$.

Let us verify that $E$ contains at least some fixed proportion of $Q$. By (3.5), we have in particular that

$$
(1-C \epsilon)|Q| \leq \operatorname{Re} \int_{Q} \nu \cdot \nabla F_{\nu} d x=\operatorname{Re}\left(\int_{E}+\int_{B_{1}}+\int_{B \backslash B_{1}}\right) .
$$

By $(2.3)(i)$, we have that

$$
\left|\int_{E} \nu \cdot \nabla F_{\nu} d x\right| \leq C|E|^{1 / 2}|Q|^{1 / 2}
$$

We wish to show that for $\epsilon$ chosen sufficiently small, and depending only on the allowable parameters, we have that $|E| \geq \eta|Q|$, for some fixed $\eta$ also having allowable dependence. We claim that it is enough to prove that

$$
\operatorname{Re} \int_{B_{1}} \nu \cdot \nabla F_{\nu} d x \leq \frac{3}{4}|Q|
$$

and that

$$
\int_{B_{2}}\left|\nabla F_{\nu}\right| d x \leq C \epsilon|Q|
$$

Indeed, given these last two estimates, and taking $\epsilon$ small enough, we obtain the desired lower bound for $|E|$, since $B \backslash B_{1} \subseteq B_{2}$. We may establish (3.8) immediately: (3.6) implies that

$$
\operatorname{Re} \int_{B_{1}} \nu \cdot \nabla F_{\nu} d x=\operatorname{Re} \sum_{Q^{\prime} \in \mathbf{S}_{1}} \int_{Q^{\prime}} \nu \cdot \nabla F_{\nu} d x \leq \frac{3}{4} \sum_{Q^{\prime} \in \mathbf{S}_{1}}\left|Q^{\prime}\right| \leq \frac{3}{4}|Q| .
$$

Next, we note that by the definition of $B_{2}$, and the weak type $(2,2)$ estimate for the dyadic maximal operator (restricted to averages over dyadic subcubes of $Q$ ), along with (2.3)(i), we have that $\left|B_{2}\right| \leq C \epsilon^{2}|Q|$. Thus, the CauchySchwarz inequality and another application of $(2.3)(\mathrm{i})$ yield (3.9).

It remains only to establish (3.2). We begin by defining, for $f \in L_{l o c}^{1}\left(\mathbb{R}^{n}\right)$, the dyadic averages

$$
A_{t} f(x) \equiv|Q(x, t)|^{-1} \int_{Q(x, t)} f(y) d y
$$


where $Q(x, t)$ denotes the minimal dyadic subcube of $Q$ containing $x$, with side length at least $t$. We set

$$
V=V(x, t) \equiv A_{t}\left(\nabla F_{\nu}\right)(x)
$$

By the geometry of $E^{*}$, and the maximality of the cubes comprising the set $B$, we have that on $E^{*}$,

$$
\operatorname{Re} \nu \cdot V \geq 3 / 4
$$

and

$$
|V| \leq(8 \epsilon)^{-1}
$$

We note that in $E^{*}$, it then follows that

$$
|(z \cdot \bar{\nu})(\nu \cdot V)| \geq \frac{3}{4}|z \cdot \bar{\nu}|
$$

for every $z \in \mathbb{C}^{n}$. Moreover, if in addition $z \in C_{\nu}$, we have that

$$
|z \cdot V| \geq|(z \cdot \bar{\nu})(\nu \cdot V)|-|(z-\nu(z \cdot \bar{\nu})) \cdot V| \geq|z \cdot \bar{\nu}|\left(\frac{3}{4}-\frac{1}{8}\right) \geq \frac{1}{2}|z|,
$$

where we have used first the triangle inequality, then the definition of the cone $C_{\nu}$ combined with the upper bound for $|V|$ in $E^{*}$, and finally the fact that $|z| \leq(1+\epsilon)|z \cdot \bar{\nu}|$ in $C_{\nu}$. Consequently,

$$
\iint_{E^{*}}\left|\gamma_{t}(x) 1_{C_{\nu}}\left(\gamma_{t}(x)\right)\right|^{2} \frac{d t}{t} d x \leq 4 \iint_{R_{Q}}\left|\gamma_{t}(x) \cdot A_{t}\left(\nabla F_{\nu}\right)(x)\right|^{2} \frac{d t}{t} d x
$$

To obtain (3.2), we need only replace the dyadic averaging operator $A_{t}$ by a nice approximate identity $P_{t}$. But we may do this easily using the uniform boundedness of $\gamma_{t}($ see $(2.2)),(2.3)(i)$, and the square function estimate

$$
\iint_{R_{Q}}\left|\left(A_{t}-P_{t}\right)(f)(x)\right|^{2} \frac{d t}{t} d x \leq C \int_{5 Q}|f|^{2} d x
$$

The latter inequality follows readily from standard orthogonality considerations (see, e.g., [4], Appendix C). We omit the routine details. This concludes the proof of our theorem. 


\section{References}

[1] P. Auscher Regularity theorems and heat kernels for elliptic operators, J. London Math. Soc. 54 (1996), pp. 890-896.

[2] P. Auscher, S. Hofmann, M. Lacey, A. McIntosh and P. Tchamitchian, The solution of the Kato square root problem for elliptic operators on $\mathbb{R}^{n}$.

[3] P. Auscher, A. McIntosh and P. Tchamitchian, Heat kernel of complex elliptic operators and applications, J. Functional Analysis, 152 (1998), pp. 22-73.

[4] P. Auscher and P. Tchamitchian, Square root problem for divergence operators and related topics, Astérisque, vol. 249 (1998), Société Mathématique de France.

[5] R. Coifman, D. Deng, and Y. Meyer, Domains de la racine carrée de certains operateurs differentials accrétifs, Annales de l'Institut Fourier 33 (1983), 123-134.

[6] R. Coifman, A. McIntosh and Y. Meyer, L'intégrale de Cauchy définit un opérateur borné sur $L^{2}$ pour les courbes lipschitziennes, Annals of Math. 116 (1982), 361-387.

[7] L. Escauriaza, unpublished communication.

[8] E. Fabes, D. Jerison and C. Kenig, Multilinear square functions and partial differential equations, Amer. J. of Math. 107 (1985), 13251367.

[9] E. Fabes, D. Jerison and C. Kenig, Necessary and sufficient conditions for absolute continuity of elliptic-harmonic measure, Annals of Math. 119 (1984), 121-141.

[10] P. Auscher, S. Hofmann, J.L. Lewis, P. Tchamitchian, Extrapolation of Carleson measures and the analyticity of Kato's square root operators, to appear, Acta Math.

[11] J.-L. Journé, Remarks on the square root problem, Pub. Math. 35 (1991), 299-321. 
[12] T. Kato, Perturbation Theory for Linear Operators, Springer-Verlag, New York 1966.

[13] T. Kato, Fractional powers of dissipative operators, J. Math. Soc. Japan 13 (1961), p. 246-274.

[14] C. Kenig and Y. Meyer, The Cauchy integral on Lipschitz curves and the square root of second order accretive operators are the same, in Recent Progress in Fourier Analysis, I. Peral, ed., North Holland Math Studies 111 (1985), 123-145.

[15] A.McIntosh, Square roots of operators and applications to hyperbolic p.d.e.'s, Miniconference on Operator Theory and PDE, Proceedings of the Centre for Mathematical Analysis, The Australian National University, Canberra, vol. 5 (1984), pp.124-136.

[16] A. McIntosh, On the Comparability of $A^{1 / 2}$ and $A^{* 1 / 2}$, Proc. Amer. Math. Soc. 32 (1972), 430-434.

[17] A. McIntosh, The square root problem for elliptic operators, Functional Analytic Methods for Partial Differential Equations, Lecture Notes in Math. vol. 1450, Springer Verlag, 1990, pp. 122-140. 